\title{
BRIGHT QUASARS AS RAPID PRECESSORS
}

\author{
Nico Roos \\ Astronomical Institute \\ Catholic University of Nijmegen \\ Toernooiveld, 6525 ED Nijmegen, The Netherlands
}

\begin{abstract}
The formation and evolution of massive binary black holes in galactic nuclei is discussed in a galaxy merger model for the activation of galactic nuclei. Mass flow into a galactic nucleus induced by the merger causes the simultaneous tightening of a preexisting binary and an increase in the fueling level of the central hole(s). The model predicts that during the final, short and most luminous phase of an AGN the precession period of the central hole will rapidly become as short as $10^{3} \mathrm{yr}$. Some observational implications are mentioned.
\end{abstract}

\section{Galaxy Interactions and Activity}

Massive accreting black holes are now widely considered to be the central engines in active galactic nuclei (AGN). On the basis of the number of quasars at earlier epochs it has been conjectured that at present most galaxies could harbour quiescent massive black holes (e.g. Blandford, 1986). In a previous paper (Roos, 1981) it was argued that mergers of galaxies with companions could be responsible for recurrent outbursts of activity in galactic nuclei by increasing the number of stars subject to tidal disruption by the central hole. The estimated merging rate seems sufficient to account for the present number of AGN (Roos, 1985a,b). The last couple of years evidence has been accummulating that AGN might be triggered by galaxy interactions (e.g. Hutchings 1987; Heckman et al. 1986; Sanders et al. 1988; Yee and Green 1987; Golombek et al., preprint). Precession of radio jets is another phenomenon related to galaxy mergers (Begelman et al. 1980, BBR), and it seems useful to investigate the possible relation between activity and precession of radio jets in the context of galaxy merger models for AGN.

\section{Massive Binary Black Holes in Normal and Active Galactic Nuclei}

Most galaxies may have undergone one or several mergers during their lifetime (e.g. Bahcall and Tremaine, 1988). If galaxies contain massive black holes then such mergers will naturally lead to the formation of massive binary black holes with separations of the order of parsecs (BBR). Further loss of orbital energy of these "wide" binaries is impeded by the depletion of stars (or gas) on orbits intersecting the binary orbit. Thus, we should perhaps assume that many, if not all, galaxies contain massive binary black holes as a result of mergers (systems of a few black holes will quickly evolve to a binary by ejecting the lighter objects).

In AGN a binary will manifest itself through precession of a jet emitted along the spin axis of the central hole. The S-symmetry observed in many radio sources and in more than 30 percent of all quasars at $z<1$ (Hutchings et 
al., 1988) might be due to the presence of such "wide" binaries. However, in many quasars the jets are strongly curved on the milliarcsec scale as well. If this curvature is also due to precession, much shorter precession periods $\left(\sim 10^{3} \mathrm{yr}\right)$, and thus smaller binary separations are required. It seems then plausible to assume that mass flow into the galactic nucleus is causing both the activity and the evolution of the central binary. In a recent paper (Roos, 1988) this hypothesis has been investigated in the case of (stellar) mass flow into the nucleus caused by a galaxy merger. The main result is that the increasing inflow rate of stars during the merger causes the simultaneous increase of activity and energy loss of the central binary. In the long early stages of activity the precession period is of order $\geq 10^{8} \mathrm{yr}$. During the short $\left(10^{4-6} \mathrm{yr}\right)$, final, most luminous phase of maximum activity (fueling rate $\sim 1 M_{\odot} \mathrm{yr}^{-1}$ ) very short precession periods of order $10^{3-6} \mathrm{yr}$ may be attained. Further evolution of the binary then proceeds rapidly via emission of gravitational waves and the central binary merges while the satellite galaxy deposits a new secondary hole in the galactic nucleus.

\section{Rapid Precession in Bright Quasars.}

The above model has some interesting observational implications: (i) quasars should evolve from weaker straight radio sources into powerful, curved sources showing the strongest curvature close to the nucleus; (ii) the relativistic material in a rapidly precessing jet will be spread over a larger range of directions than in a straight jet and will interact more strongly with the ambient medium.

There are some observational indications that powerful quasars do exhibit the kind of properties sketched above: (i) jets in many compact steep-spectrum quasars appear to precess rapidly and interact strongly with the ambient medium (van Breugel et al. 1988). Simple unified schemes based on the relativistic beaming hypothesis (Orr and Browne, 1982) fail when applied to powerful quasars, because (ii) superluminal motion is observed in too many sources and (iii) core-dominated sources have intrinsically stronger curvature than other sources (De Bruyn and Schilizzi, 1986), and this curvature sets in already at a small distance from the core (Hooimeyer and Roos, in preparation). It is tempting to speculate that the high incidence of bright cores and superluminal motion in powerful quasars is related to the strong, small-scale intrinsic curvature that may be due to rapid precession of the central engine.

\section{References.}

Bahcall, S. and Tremaine, S., 1988, Ap.J. (Letters), 326, L1

Begelman M.C., Blandford, R.D. and Rees, M.J. 1980, Nature 287, 307.

Blandford, R.D., 1986 in Superluminal Sources, eds. Zensus \& Pearson, p310.

De Bruyn, A.G. and Schilizzi, R.T., 1986, in IAU Symp.119, Quasars, p203

Heckman, T.M., Smith, E.P., Baum S.A. et al., 1986, Ap.J. 311, 526.

Hutchings, J.B., 1987, Ap.J. 320, 122.

Hutchings, J.B., Price, R. and Gower, A.C., 1988, Ap.J. 329, 122.

Orr, M.J.L. and Browne, I.W.A., 1982, M.N.R.A.S. 200, 1067

Rees, M.J., 1984, Ann. Rev. Astron. and Astroph. 22, 471.

Roos, N. 1981, Astron. and Astroph. 104, 218.

Roos, N. 1985a and b, Ap.J. 294, 479 and 486.

Roos, N. 1988, Ap.J., in press.

Sanders, D.B., Soifer, B.T., Elias, J.H. et al. , 1988, Ap.J. 325, 74.

van Breugel, W. et al. 1988, in IAU Symp. 129, The Impact of VLBI etc., p115.

Yee, H.K.C. and Green, R.F. 1987, Ap.J. 319, 28. 


\section{DISCUSSION}

GASKELL I have proposed that double peaked BLR profiles are a consequence of seeing two BLRs - one associated with each of the black holes in your "wide" case. The best cases tend to be objects with well-defined large scale jets. This could fit in with what you have just said but another explanation could be an orientation effect.

ROOS Yes, I agree, that is an interesting possibility, especially since broad line clouds seem to move at small distances of the central massive object. However, the orbital velocity of a secondary hole in a wide binary has an orbital velocity of only $500 \mathrm{~km} \mathrm{~s}^{-1}$ at the estimated separation of one tenth of the cusp radius.

PENSTON If you have a binary nucleus you will not just get double peaked BLRs, but you will also get a third peak from low velocity gas in a common envelope.

GASKELL That's right, and I think it might well be what is being observed in many cases (although it is confused with the NLR).

NORRIS Have you looked for any relationship between the period of precession or orbital motion and the period of repeated starburst activity?

ROOS I should stress that in this merger model the nucleus becomes active when the two nuclei are already quite close together, i.e., separations smaller than $\sim 1$ kpc. So starbursts induced by the merger would precede activity (or rapid precession) of the central engine. 